\title{
Study of Awareness, Understanding and Attitude of parents of Children (1-12 yrs of age) with Epilepsy
}

\author{
G. P. Kaushal, ${ }^{1}$ S B Shrivastva, ${ }^{2}$ Monideepa Dutta ${ }^{3}$ \\ ${ }^{1}$ In- charge Neonatal Unit, Department of Pediatrics Dr. Baba Saheb Ambedkar Medical College and Hospital, Rohini, Delhi, \\ ${ }^{2} \mathrm{HOD}$, Department of Dermatology, Dr. Baba Saheb Ambedkar Medical College and Hospital, Rohini, Delhi, ${ }^{3}$ Junior Consultant \\ (PICU), AMRI Hospital, Kolkata, India
}

Corresponding author: Dr. S. B. Shrivastava, 55 A, Sunder Apartments, GH 10, Pachim Vihar, Delhi 110087, India

DOI: http://dx.doi.org/10.21276/ijcmsr.2018.3.4.34

How to cite this article: G. P. Kaushal, S B Shrivastva, Monideepa Dutta. Study of awareness, understanding and attitude of parents of children (1-12 yrs of age) with epilepsy. International Journal of Contemporary Medicine Surgery and Radiology. 2018;3(4):D150-D156.

\section{A B S T R A C T}

Introduction: Epilepsy is a highly stigmatizing disease in the developing countries, associated with misconception and discrimination. Studies on general public regarding epilepsy have been carried out in many countries, but there are very few studies regarding awareness, understanding and attitude of parents of children of epilepsy toward epilepsy. Study aimed to obtain a quantitative measurement of the parental awareness, understanding and attitude toward epilepsy, and used univariate analysis to identify individual predictive variables.

Material and Methods: It was a prospective study conducted on parents of 100 consecutive children of Epilepsy, who were enrolled in the Seizure Clinic of Dr. Baba Saheb Ambedkar Hospital, multispecialty, post-graduate teaching hospital of Govt. of Delhi. Structured questionnaire were provided to the parents, their answers/ data tabulated and statistical analysis were performed with SPSS Version 15.0 package. Chi-square test was used to examine the association between the responses and socio-economic and other variables. The $\mathrm{P}$ value 0.05 was considered as the cut off value for significance.

Results: $82 \%$ parents heard about epilepsy but awareness regarding personal acquaintance with an epileptic person or witnessing an epileptic seizure was recorded in only $34 \%$ and $39 \%$ cases respectively. No association was found between awareness and socio-economic variables like education of parents, family income and area of residence $(p$ value $>0.05)$. A poor understanding of epilepsy as a disease was found among the parents who tended to equate epilepsy to mental retardation (33\%) and to insanity (28\%). Parent's education and family income were important determinants of these false beliefs of equalizing epilepsy with mental retardation and insanity ( $P$ value 0.013 and 0.028 respectively). Knowledge regarding cause of epilepsy was fair as $40 \%$ of parents correctly mentioned nervous system disorder as a cause for epilepsy. $84 \%$ parents believed epilepsy is a curable disease by medicines. Education of father was significantly associated with the knowledge ( $P$ value 0.042 ) and that of mother with the cure of epilepsy ( $P$ value 0.012 ).

Conclusion: Statistically significant strong association between attitude and understanding regarding cause and cure of epilepsy ( $p$ value 0.010 and 0.000 respectively) was found among parents in this study.

Key words: Epilepsy, Seizure, Attitude of Parents of Epilepsy Children

\section{INTRODUCTION}

Epilepsy is a highly stigmatizing disease in developing countries and may rank with leprosy and mental illness in this respect. ${ }^{1}$ It is well documented that knowledge is a vital factor in the ability to cope successfully with epilepsy. Misconception and deficit in knowledge have implications, not only for psychosocial well being but also for medical compliance. ${ }^{2}$ The social stigma and the discrimination against epileptic are often more devastating and harmful than the disease itself. ${ }^{3}$ If these problems are to be dealt with, a systematic study of awareness, understanding and attitude toward epilepsy is the necessary first step. Studies on general public regarding this have been carried out in many countries throughout the world. ${ }^{4,5,6}$ Some of these earlier studies have tried to identify socio-economic variables associated with negative attitudes toward people with epilepsy but there are very few studies regarding awareness, understanding and attitude of parents of children of epilepsy toward epilepsy. The present study analyzed clinico-etiological and socioeconomic profile of patients with epilepsy and also introduces a novel approach to obtain a quantitative measurement of the parental awareness, understanding and attitude toward epilepsy, and used univariate analysis to identify individual predictive variables. The basis of involving parents is because of their special role in upbringing of these epileptic children and thus finding their knowledge and attitude to epilepsy is of utmost importance, so that we can design information campaigns to fill up the deficits or gaps. Study aimed to obtain a quantitative measurement of the parental awareness, understanding and attitude toward epilepsy, and used univariate analysis to identify individual predictive variables. 


\section{MATERIAL AND METHODS}

It was a prospective study conducted on 100 consecutive children between the age group of 1 to 12 years and their parents enrolled in the seizure clinic of Dr. B. S. A. Hospital, Rohini, Delhi from January 2009 onwards. Subjects were selected after matching the under mentioned inclusion and exclusion criteria from those who agreed to take part in this study.

\section{Inclusion criteria}

a. Child having 2 or more seizure episodes more than 24 hours apart and

b. Child on anticonvulsant therapy at the time of study

c. Child with EEG and Neuroimaging (CECT) done

d. Child with parents who can give a coherent history.

\section{Exclusion criteria}

Child with

a History of typical febrile convulsion.

b Acute infective state.

c Seizures after any medication or toxins.

d Congenital heart disease.

e Any other gross congenital anomalies.

f Any neurological deficits.

g Whose parents cannot give coherent history.

A proforma was prepared to collect the clinico- etiological and socio-economic data of the patients. These includes name, age, sex, etiological diagnosis, type of seizure, drug history, family history including family education and family income, area of residence, immigration and health care facilities availed. EEG and CECT reports were recorded in all cases. Education of parents was divided into four categories: illiterate, primary, secondary and more than secondary. We categorized the parents of the patients according to their monthly income i.e. low income group (less than 5,000 / month), middle income group $(5,000$ to $20,000 /$ month $)$ and

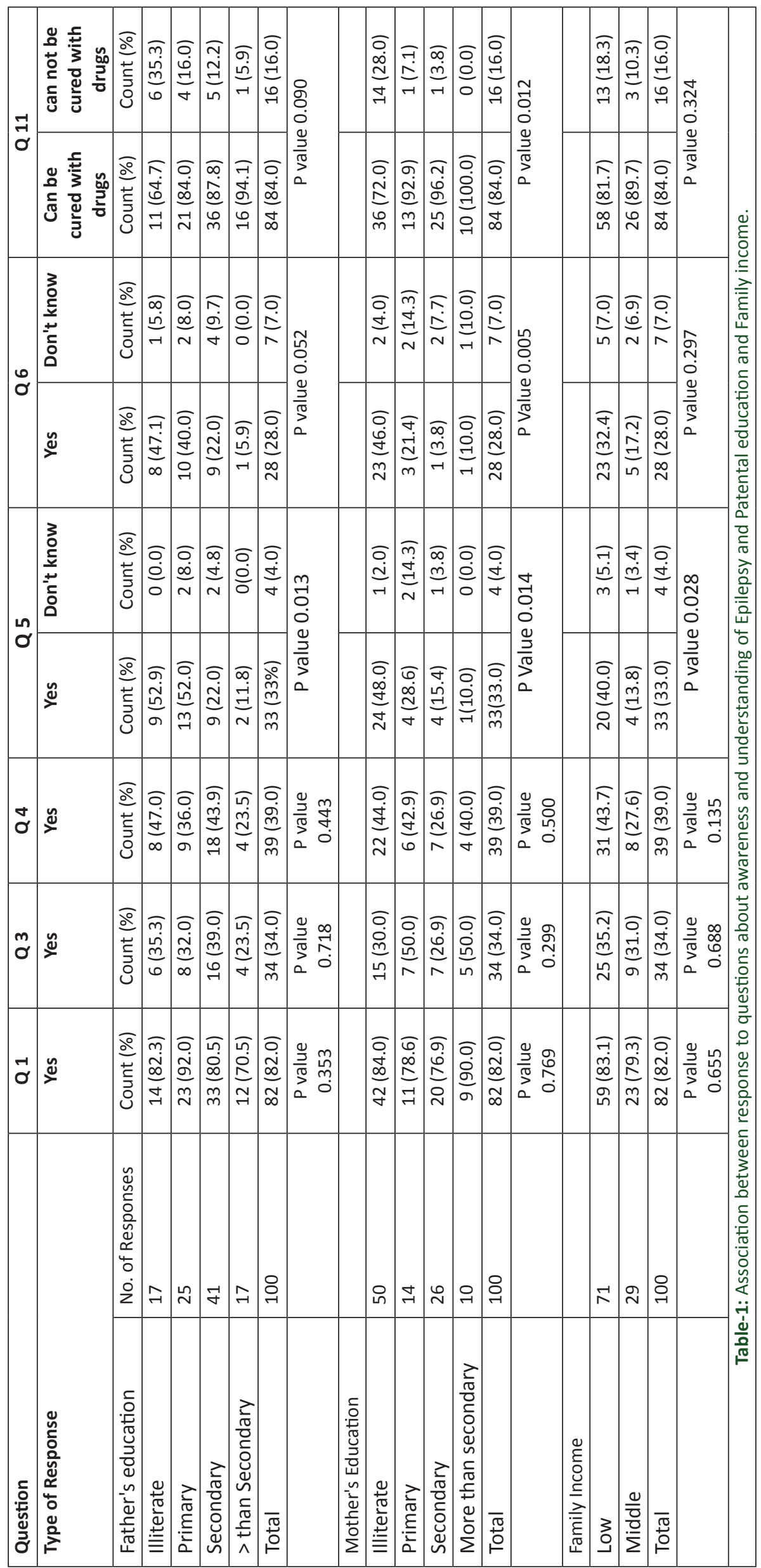


high income group (more than 20,000 /month). ${ }^{3}$ Area of residence was grouped into three categories viz. J-J Cluster, rural and urban. Thereafter the parents were interviewed regarding Awareness, Understanding and Attitude towards epilepsy through Structured questionnaire, and their answers were recorded in the under mentioned proforma. The questionnaire investigated three major issues: Familiarity with epilepsy before the child suffered from the same (Q1-Q4), Understanding of epilepsy (Q5-Q11) and Attitude of parents toward the patient with epilepsy (Q12-Q18).

The questions regarding attitude toward epilepsy allowed for selection between four graded answers with the first choice always reflecting minimal rejection toward a patient with epilepsy and the last choice reflecting the maximum rejection. Points were assigned for each possible answer to the attitude related questions Q12-Q18 (1 point for answer 1, 2 points for answer 2, etc), and by adding them up a number was obtained that reflect the overall or mean attitude of each interviewee toward patient with epilepsy. On this quantitative "scale of Parental Rejection" a value of 7 reflected the least degree of rejection and the value of 28 reflected the maximal degree of rejection.

\section{STATISTICAL ANALYSIS}

Descriptive data was tabulated, presented as absolute numbers and percentage (\%). Statistical analyses were performed with the SPSS version 15.0 package. Chi-square test was used to examine the association between the responses and the socio-economic and all other variables in univariate analysis. A 5\% level of significance was used. The $\mathrm{p}$ value $<0.05$ was considered as the cut off value for significance. Informed consent of the parents was taken before proceeding for the study. The study was approved by ethical committee of the institute.

\section{RESULTS}

Table 1 shows that father of $17 \%$ of children were illiterate and $17 \%$ were educated beyond secondary level education. Significant number of mother was illiterate $(50 \%)$ and only $10 \%$ of them had education level more

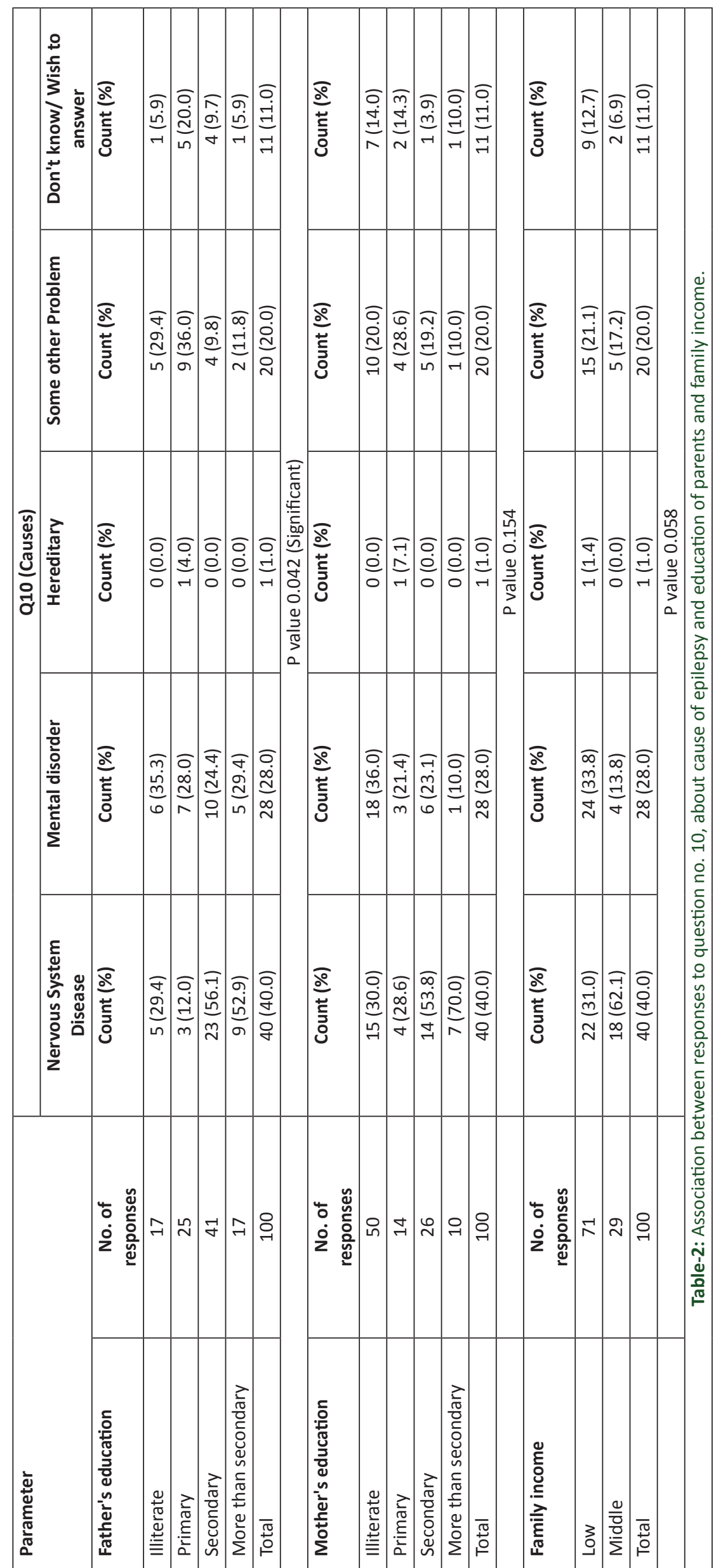



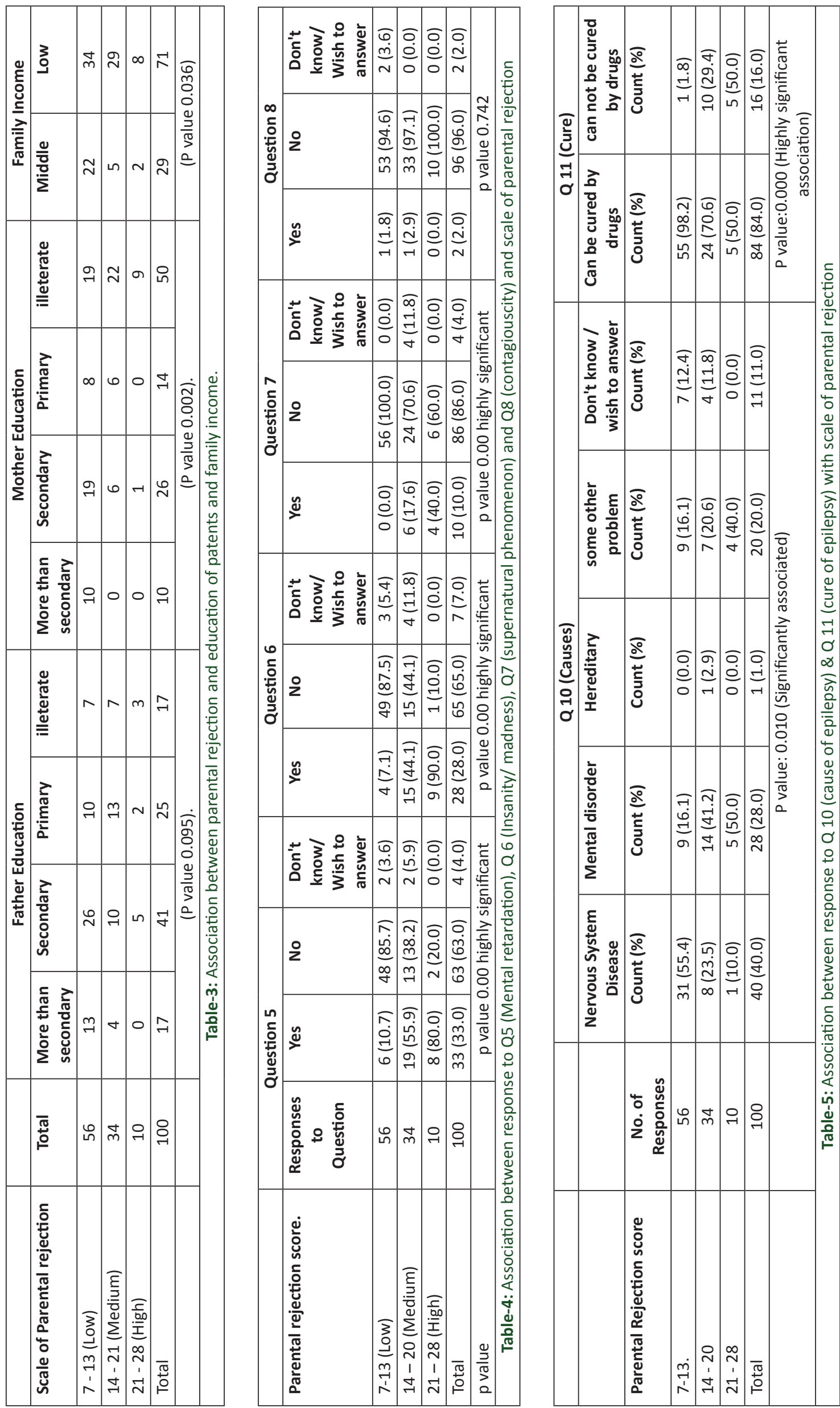
than secondary. $71 \%$ and $29 \%$ of cases were from low and middle income group respectively and no cases were from high income group. $33 \%$ and $28 \%$ of parents attributed epilepsy to be a type of mental retardation and insanity respectively. Parents' education and family income were important determinants of these false beliefs of equalizing epilepsy with mental retardation and insanity ( $P$ value 0.013 and 0.028$) .84 \%$ parents believed epilepsy as a curable disease by medicines.

Table 2. shows that $40 \%$ of parents correctly mentioned nervous system disorder as a cause for epilepsy. Education of father was significantly associated with the knowledge about cause of epilepsy.( $P$ value 0.042$)$. Table 3 shows that $56 \%$ parents had low score on parental rejection scale while $34 \%$ had score between 14-21. Only 10\% cases revealed high degree of rejection (Score 21-28). among 56 cases having low score of parental rejection, 7 had illiterate father. No father had education more than secondary among 10 parents who revealed high degree of rejection. No association found between scale of parental rejection and father's education ( $p$ value 0.095 ). it further shows that among 56 cases having low score of parental rejection, 19 had illiterate mother and 29 had mother's education more than primary. No mother had education more than secondary among 10 parents who revealed high degree of rejection; and 9, out of these 10 were illiterate. Scale of parental rejection and mother's education significantly associated ( $p$ value 0.002 ). the table also reveals that 8 , out of $10(80 \%)$ parents who scored high on rejection scale, were from low income group; while proportion of parents with low family income decreased (34 of 56 i.e.60.7\%) with low score on scale. Family income found to be significant negative predictive factor ( $\mathrm{P}$ value 0.036$)$. As per Table 4 the false beliefs that epilepsy 'is a type of mental retardation' ( $p$ value 0.000 ), 'is a type of insanity' ( $p$ value 0.000 ) 'caused by super natural phenomenon or evil spirits'( $p$ value 0.000$)$ and 'has a seasonal variation'( $p$ value 0.011 ) were the important contributive factors to the negative attitude of the parents. Table 5 indicates that significant strong association between attitude and understandings about cause and cure of epilepsy ( $p$ value 0.010 and 0.000 respectively) was found in this study.

\section{DISCUSSION}

Awareness and Understanding of Parents

The study showed (Table no. 1) that majority of parents (82\%) heard about epilepsy before their child had the same, which is comparable with other study on parents ${ }^{7}$ but slightly less as compared to general public as revealed by several studies in different countries. ${ }^{4,8,9}$ Awareness of parents was far less in issues like personal acquaintance with an epileptic person (34\%) or witnessing an epileptic seizure (39\%). ${ }^{10,11,9}$ This may be due to the fact that the parents were young enough to have those kinds of experiences. No association was found between awareness and socio-economic variables like education of parents, family income and area of residence ( $p$ value >0.05). When understanding of epilepsy was evaluated, it was found that parents thought in more negative ways in some matters like equalizing epilepsy with mental retardation and insanity. ${ }^{8,10,11,5}$ but at the same time showed less prejudices than general public by denying contagiousness of or any super natural association with epilepsy. ${ }^{12}$

The idea of epilepsy as a type of mental retardation ( $p$ value 0.013 and 0.014 ) and insanity ( $p$ value 0.05 and 0.005 ) was significantly associated with parents' (father's and mother's respectively) education; those with higher level of education thought it more appropriately. $40 \%$ of respondents from low income group as compared to $13.8 \%$ of middle income group thought, epilepsy as a type of mental retardation ( $p$ value 0.028). As it is known that parents coming from lower income group are very little aware of education of their children and may be thus think epilepsy is a type of mental retardation to find an easier explanation for their children's poor school performance rather than spending times to look for other factors. Regarding contagiousness of epilepsy previous studies had pointed out that people with epilepsy considered their disease less infectious as compared to nonepileptic people. It was very obvious for parents to support non contagiousness of epilepsy as they were in close contact with their child and even not getting infected to have a seizure.

The perceived causes of epilepsy (Table 2) as told by parents in this present study were much different from those stated in earlier studies, either by parents. ${ }^{13}$ or by general public. ${ }^{12,9}$ It was good to see that $40 \%$ of respondents in the present study correctly said epilepsy to be a nervous system disorder which was much higher in comparison to earlier studies but significant no of parents (31\%) were uncertain about the causes while $28 \%$ thought it is due to some mental or psychological problem. As it was seen from other studies that higher level of education influenced the answer of respondents in a favorable way. ${ }^{4,5}$ Present study also evidenced significant association between higher level of father's education and correct answer ( $p$ value 0.042 ).

When parents were asked that what they thought about cure of epilepsy, an affirmative answer was given by $84 \%$, while the rest believed to the contrary, that either it could not be cured by drugs or cured by its natural course. It was expected that these parents would give a more positive response toward cure by drugs than general public ${ }^{5}$, as this study was conducted on parents who came for taking treatment for their children. Again this positive response regarding treatment of epilepsy with drug was strongly associated with mother's education ( $p$ value 0.012 ).

\section{Attitude toward Epilepsy}

The overall attitude of parents as measured by this scale seemed to be positive with only $10 \%$ parents showed high rejection (score between $21-28$ ), and more than half of the respondents $(56 \%)$ revealed to have low rejection (score between 7 - 13) toward epilepsy (Table 3). This result was in contrary to the various studies in different parts of India ${ }^{12,11,4}$ and world. ${ }^{14,10,9,6,3}$ which investigated attitude toward epilepsy not only among general public but also in school teachers. Bains and Raizada et al. ${ }^{13}$ in their study in Punjab found that $57 \%$ of parents irrespective of religion, literacy level, and rural/ urban distribution viewed epilepsy as a social stigma and wanted to keep it as a secret. These results however must be interpreted with reasonable caution because of the inherent problem in all studies of this type, namely, the problem of the potential tendency of some 
people to answer questions reflecting their social attitudes in a "socially desirable" or "politically correct" manner that does not always represent their most sincere thoughts.

In this study a number of factors were found to be associated with higher tendency of rejection like low level of education in mother ( $p$ value 0.002 ), low family income ( $p$ value 0.036 ) and residence in J-J cluster (0.008). Similarly study carried out in Austria also identified low socio-economic background as a negative predictive factor for attitude toward epilepsy. ${ }^{15}$ For the purpose of elucidating major false beliefs about epilepsy that have contributed to negative attitude toward epilepsy, the relationship between the understanding of, and attitude toward epilepsy of the respondents was analyzed (Table 4). Five items of understanding and beliefs regarding cause and cure of epilepsy were selected for finding association with score of rejection scale. Among these, score of rejection was strongly associated with the beliefs that epilepsy is a type of mental retardation ( $p$ value 0.000 ), type of insanity ( $p$ value 0.000 ), caused by super natural phenomenon ( $p$ value 0.000 ). Statistically significant association was also found between score of rejection and the idea that epilepsy has a seasonal variation ( $p$ value 0.011 ). Comparatively more parents' with high score (70\%) on rejection scale thought that epilepsy has a seasonal variation than parents' with a low score (19.6\%) (Table 5).

Kim et al. ${ }^{9}$ in their study found that most probable contributive factor in forming negative attitude toward epilepsy was 'epilepsy cannot be cured' followed by 'epilepsy is caused by mental illness. In this study we also found similar type of statistically significant strong association between attitude and beliefs regarding cause and cure of epilepsy ( $p$ value 0.010 and 0.000 respectively). Parents who thought mental problem as a cause of epilepsy or who were unaware of the cause, seemed to possess more negative attitude. Similarly those parents showed more positive attitude who believed cure is possible with drugs. Study in Austria also found in a comparable way that little knowledge about epilepsy, misconception of epilepsy as a form of insanity, independently predisposed to unfavorable attitude toward epilepsy. ${ }^{15}$

\section{CONCLUSION}

Statistically significant strong association between attitude and understanding regarding cause and cure of epilepsy ( $p$ value 0.010 and 0.000 respectively) was found among parents in this study. Low level of education and low family income are the important determinants of this inappropriate knowledge.

\section{REFERENCES}

1. Rwiza HT, Matuja WBP, Kilonzo GP, Haule J, Mbena P, Mwang'ombola R, Jilek-Aall L. Knowledge, attitude, and practice toward epilepsy among rural Tanzanian residents. Epilepsia 1993; 34(1): 1017-1023.

2. Galletti F, Gabriella SM. Counseling children and parents about epilepsy. Patient Education and Counseling 2004; 55(3): 422-5.

3. Anup KT, Anand MV, Ram JI, Prolima T, Pragya M. Knowledge awareness and attitude about epilepsy among schoolteachers in India. Seizure 2008; 4(5): 1-7.

4. Radhakrishnan K, Pandian JD, Santhoshkumar T, Thomas SV, Deetha TD, Sarma PS, Jayachandran D, Mohamed E. Prevalence, knowledge, attitude, and practice of epilepsy in Kerala, South India. Epilepsia 2000;41(3): 1027-1035.

5. Diamantopoulos N, Kaleyias J,Tzoufi M, Kotsalis C. A survey of public awareness, understanding, and attitudes toward epilepsy in Greece. Epilepsia 2006;47(12): 21542164.

6. Jacoby A, Gorry J, Gamble C, Baker GA. Public knowledge, private grief: a study of public attitudes to epilepsy in the United and implications for stigma. Epilepsia 2004;45(2): 1405-1415.

7. Center Disease Control and Prevention. Current Trends Prevalence of Self-Reported Epilepsy. United States, 1986-1990. Retrieved from http://www.cdc.gov/mmwr/ preview/mmwrhtml/00033483.htm

8. Caveness WF, Gallup GH Jr. A survey of public attitudes toward epilepsy in 1979 with an indication of trends over past thirty years. Epilepsia 1980;21(4): 509-518.

9. Kim MK, Kim IK, Kim BC et al. Positive trends of public attitudes toward epilepsy after public education campaign among rural Korean residents. J Korean Med Sci 2003;18(5): 248-254.

10. Canger R, Cornaggia C. Public attitudes toward epilepsy in Italy; result of a survey and comparison with U.S.A. and West German data. Epilepsia 1985;26(4): 221-226.

11. Gambhir SK, Kumar V, Singhi PD, Goel RC. Public awareness, understanding and attitudes toward epilepsy. Indian J Med Res 1995;102(1): 34-38.

12. Sureka RK, Sureka R. Knowledge, attitude and practices with regard to epilepsy in rural north-west India. Annals of Indian Academy of Neurology 2007;10(3):160-164.

13. Bains HS, Raizada N. parental attitude towards epilepsy. Indian paediatrics 1992; 29(5): 1487- 1490.

14. Finke M. Public attitudes toward epilepsy in the Federal Republic of Germany: trends over the past thirty years. Epilepsia1980; 21(4): 201.

15. Spatt J, Bauer G, Baumgartner C, Feucht M, Graf M, Mamoli B, Trinka E. Predictors for negative attitudes toward subjects with epilepsy: A representative survey in general public in Austria. Epilepsia 2005; 46(5):736742 .

Source of Support: Nil; Conflict of Interest: None

Submitted: 22-11-2018; Accepted: 15-12-2018; Published online: 02-01-2019 


\section{Proforma for study of awareness, understanding and attitude toward epilepsy:}

1. Have you ever heard of a disease called epilepsy before your child has the same?

2. Do you have a close relative with epilepsy other than this child?

3. Do you personally know someone with epilepsy other than this child?

4. Have you ever seen an epilepsy before your child has an attack?

5. Do you think epilepsy is a type of Mental Retardation?

6. Do you think epilepsy is a type of insanity or madness?

7. Do you think epilepsy is caused by super natural phenomena such as evil spirits, the Devil, the moon cycle etc.

8. Do you think epilepsy is contagious or affect other children of your family?

9. Do you think epilepsy has seasonal variation?

10. What do you think is the cause(s) of epilepsy? (You may choose more than one answer)
a. A nervous system disorder
b. A congenital abnormality
c. A mental or psychological disorder
d. A hereditary disease
e. A blood disease
f. Some other problem
g. I don't know or wish to answer

11. In your view how epilepsy can be cured
a. By drugs
b. Not by drugs/ cured by natural way
c. By supernatural ways
d. I don't know or wish to answer

12. How you reacted when you learned that your child has epilepsy?
a. I treated him / her as before.
b. I felt a little awkward and afraid.
c. I felt very awkward and afraid.
d. I don't know or wish to answer.

13. Have you disclosed or wanted to disclose that your child is having epilepsy?
a. I talk about it freely with any one.
b. I talk about it only to people very close to me.
c. I keep it as a secret from everybody.
d. I don't know or wish to answer.

14. Do you like to associate his / her condition with some social situation?
a. Never
b. Sometimes
c. Definitely
d. I don't know or wish to answer.

15. According to you how is the relationship with other siblings or schoolmates before or after the epilepsy?
a. They treat him / her as before.
b. They are little hesitant in their behaviour.
c. They are very much hesitant in their behaviour.
d. I don't know or wish to answer.

16. According to you how school performance has been affected by epilepsy?
a. Not affected.
b Affected a little.
c. Affected very much.
d. I don't know or wish to answer.

17. According to you how important it is to stick to prescribed treatment?
a. Very much necessary, I don't stop any of them
b. Can be stopped for some time
c. Not so important
d. I don't know or wish to answer

18. Do you think that your child is going to be normal after completion of advised treatment?
a. Absolutely, he / she will be completely normal
b. Not completely normal
c. Not at all
d. I don't know or wish to answer 